\title{
BH4 Domain
}

National Cancer Institute

\section{Source}

National Cancer Institute. BH4 Domain. NCI Thesaurus. Code C13915.

$\mathrm{BH} 4$ is present only in the amino-terminal regions of pro-survival family bcl-2 members and is essential for the anti-apoptotic function, although deletion of $\mathrm{BH} 4$ does not necessarily impair the ability of $\mathrm{BH} 4$-containing proteins to bind to a variety of other bcl2-related proteins. 